\title{
Design of a Platform for Teaching Embedded Systems Engineering
}

\author{
Ivan Kastelan, Miodrag Temerinac \\ University of Novi Sad \\ Faculty of Technical Sciences \\ Novi Sad, Serbia \\ e-mail: ivan.kastelan@ieee.org
}

\author{
Nikola Teslic \\ RT-RK Institute for Computer Based Systems \\ Novi Sad, Serbia \\ e-mail: nikola.teslic@rt-rk.com
}

\begin{abstract}
This paper presents the design of a platform for teaching majority of courses in the embedded computer engineering curriculum. The learning platform is a result of the E2LP project whose main idea is to provide a unified platform which will cover a complete process for embedded computer systems learning. The main body of the platform is the base board with FPGA connected to a wide range of interfaces audio, video, communication, memory and user I/O. Additionally the base board can be connected to one extension board via the standardized Mezzanine interface. Extension boards broaden the range of applications of the platform beyond digital system design and allow the platform to be used in the entire computer engineering curriculum. The first application of the platform in laboratory exercises has shown a positive acceptance of the platform by students.
\end{abstract}

\section{INTRODUCTION}

$\mathrm{A}_{\mathrm{i}}^{\mathrm{s}}$ $\mathrm{S}$ embedded systems are becoming more complex, the industry requires embedded system engineers to tackle increasingly complex problems and to be able not only to solve basic everyday issues in system design, but also to envision and suggest new solutions and systems in order to achieve a specific task. This increasing complexity in the industry requires a significant change in the education of future embedded system engineers. E2LP project [1]-[3] aims to bring that change with its usability in the whole curriculum and with its elimination of the overhead in teaching.

Increased role of computer based embedded systems in various industrial applications has produced a growing need for embedded system engineers. As of today, the job market is very competitive for highly qualified electronics systems engineers [4]. Furthermore, the European Centre for the Development of Vocational Training in its publication "Skills supply and demand in Europe, Medium-term forecast up to 2020" [5] predicts that increase of $10.7 \%$ in new engineering positions across all disciplines in Europe will be reached up to 2020, compared to the 2010 level. Similar projections are available for USA [6] and Asia [7]. Consequently, many technical faculties have put more

The research leading to these results has received funding from the European Union's Seventh Framework Programme (FP7/2007-2013) under grant agreement $\mathrm{n}^{\circ} 317882$. emphasis on embedded systems learning by introducing a number of active learning laboratory-based courses [8].

The approach in E2LP project targets the lab education efficiency with the idea to use a single comprehensive platform for the complete curriculum. The main intention is to make the educational process more efficient and to introduce more interaction between the education and further embedded system research and development, which facilitates an optimal solution for a specific problem. The main E2LP objective is to efficiently educate future engineers capable of coping with current challenges in realtime embedded computer engineering field. It will further provide a learning environment that moves focus from hardware to software and encourages learning of the embedded systems, but without compromising knowledge related to the hardware design.

This paper describes the hardware design of a platform which is the result of E2LP project. The platform in its current form can be used for teaching the majority of embedded engineering curriculum at the university level. Due to its increased modularity, the platform is easily extendable in order to provide support for an even larger set of courses, spanning outside of the range of embedded engineering courses into the fields of electrical engineering and systems control, to name a few.

The paper describes the electrical and mechanical design of E2LP Base Board based on Field Programmable Gate Array (FPGA) and two of its extension boards connected to it through Mezzanine interface. The Base Board and its Mezzanine extension boards are intended to provide the unified platform for the complete embedded system engineering curriculum.

This paper is organized as follows: after the introduction, section 2 gives a brief overview of the E2LP platform. Section 3 describes in more detail the hardware of E2LP system - Base Board and two extension boards. Section 4 gives a very brief summary of the software support for E2LP platform. Finally, section 5 gives some concluding remarks. 


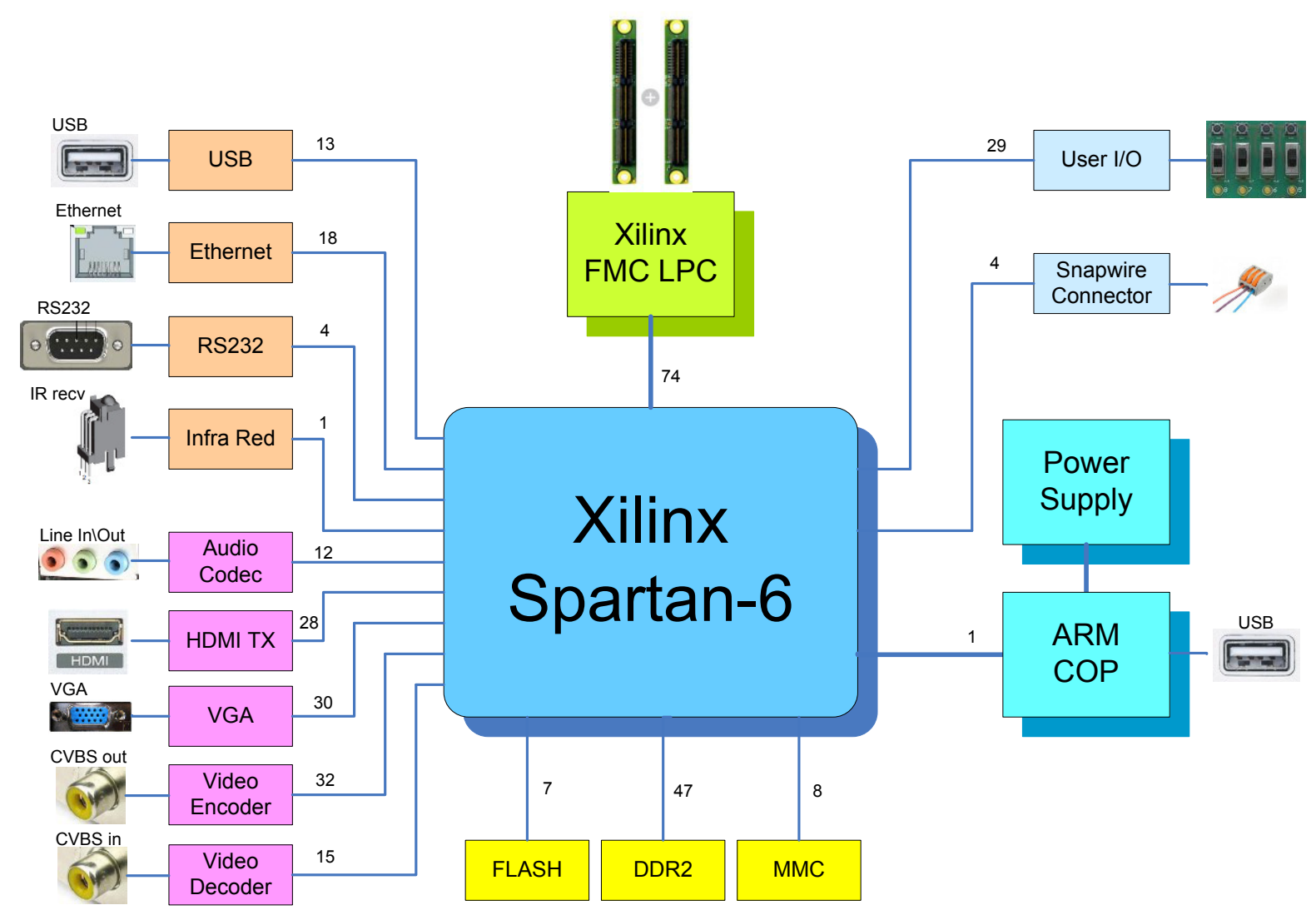

Figure 1. E2LP Base Board

\section{PLATFORM OVERVIEW}

The embedded engineering learning platform [1] is based on FPGA which is connected to various interfaces and peripherals on the platform. It is intended to be used in multiple courses in the curriculum of computer engineering. The base board of the platform supports teaching courses in digital and computer system design, using FPGA for implementation of the designed digital systems.

Through its mezzanine connectors, the base board communicates with extension boards which support other courses in the curriculum, e.g. an extension board with a digital signal processor (DSP) supports courses in signal processing. With this approach the platform aims to cover the most of the computer engineering curriculum reducing the time students spend adjusting to different platforms and tools. Instead, the students have more time to learn important topics in relevant subjects in computer engineering.

\section{E2LP PLATFORM HARDWARE}

This section explains in more detail the hardware of the components of E2LP platform:

1. Base Board;

2. Extension board based on ARMADA processor;

3. Extension board based on a simpler ARM processor.

\section{A. Base Board}

The E2LP Base Board performs the following functions:

- based on FPGA, provides the central point of the E2LP platform on which all other parts are connected;

- $\quad$ supplies power for the whole E2LP platform;

- controls programming the FPGA and Central Processing Units (CPUs) on extension boards;

- provides a basic user interface;

- provides storage, multimedia and communication interfaces for the platform;

- provides the platform for digital system design;

- provides test points for debugging.

The block diagram (Fig. 1) gives a high level overview of the E2LP Base Board.

The key building modules of the E2LP Base Board are:

- Xilinx Spartan-6 FPGA;

- ARM-based control processor;

- Mezzanine connector to extension board (Xilinx FMC LPC standard);

- DDR2, flash and multimedia card memory;

- user interface (8 switches, 6 buttons, 8 LEDs, alphanumeric LCD screen);

- $\quad$ snapwire connector (8 pins);

- CVBS video encoder and decoder;

- $\quad$ video output (VGA, HDMI); 
- audio sub-system;

- communication interfaces (USB, Ethernet, RS232 and Infra-red).

FPGA is directly connected to all interfaces on the Base Board, as well as the Mezzanine connector which allows its connection to external extension boards. It is the central point of the platform and supports teaching digital system design, computer system design, FPGA accelerated computing, reconfigurable systems, System-on-Chip design, hardware/software co-design and design of multimedia and communication systems.

The choice of interfaces for the Base Board was decided based on the extensive study of teachers' and students' requirements which was conducted at the start of E2LP project. Investigation of learning models showed that E2LP platform should aim at satisfying learning requirements of the four major types of learners: convergers, divergers, accommodators and assimilators. It should also aim at satisfying needs of visual, auditory and tactile learners.

Teachers' and students' requirements were collected by conducting a study which involved surveys and data analysis. Teachers' and students' questionnaires revealed the needs for both groups from the functional and technical point of view. After careful analysis of the results of this study, the final list of interfaces was compiled taking care of the limitations of the chosen FPGA.

Fig. 2 presents the 3D overview of the base board. The board is enclosed in the box which has a transparent cover. The cover is removable and consists of two parts: a smaller and a larger cover. The following are the three use cases of the board, with respect to the state of the covers:
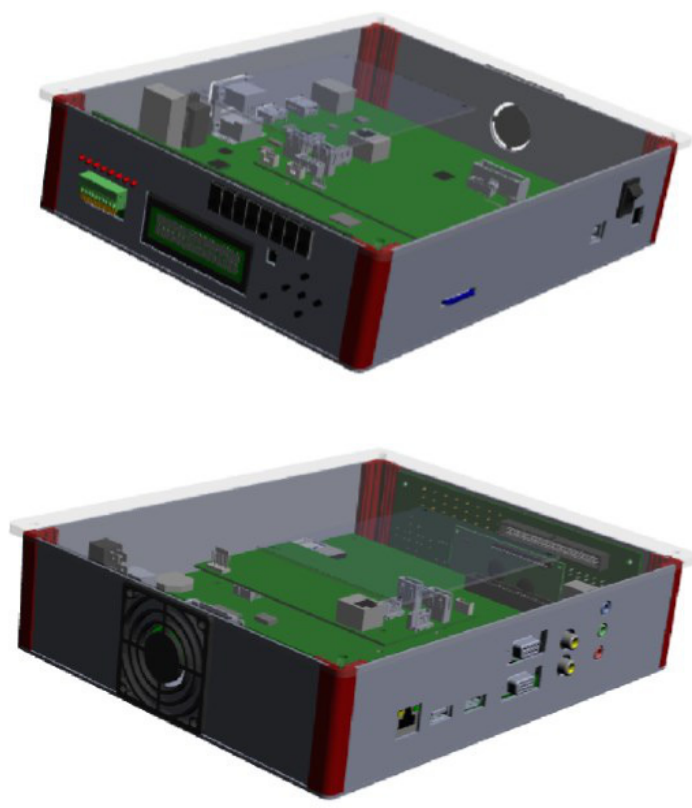

Figure 2.3D view of the base board
- both covers closed - suitable when the user wants to protect the board from external influences and still be able to see the contents on the board through a transparent cover; this case can be used with the Base Board only, or with the extension board which does not require cables;

- small cover open, large cover closed - suitable when the user wants to protect the board from external influences, but the extension board requires external connection with cables, which go through the open small cover;

- both covers open - suitable when the user wants to have physical access to all components of the board.

JTAG chain is used to configure FPGA, Complex Programmable Logic Device (CPLD) and Platform Flash on the E2LP base board. CPLD is used as a central router of the JTAG chain on the board. The board supports three configuration cases:

- Platform Cable - Platform Flash - CPLD - FPGA: In this configuration, the Xilinx Platform Cable is used to configure the three JTAG components on the board; this case is a required starting point for board configuration during the board bring-up, which configures the CPLD; this case can also be used whenever the configuration of Platform Flash and FPGA is performed using the Platform Cable;

- COP - Platform Flash - FPGA: This configuration is triggered by the control processor (COP) on the board. The COP sends the signal to the CPLD to reroute the JTAG chain such that the COP becomes the JTAG master. COP can then configure both Platform Flash and FPGA on the board. This is the suggested default configuration case since the E2LP software can be used to configure the FPGA on the board;

- COP - Platform Flash - FPGA - Mezzanine: This configuration is triggered by the Mezzanine board. When attached, the signal bit informs the CPLD to re-route JTAG chain such that the COP becomes the JTAG master and can configure Platform Flash, FPGA and Mezzanine extension board, if the JTAGconfigurable component exists on the extension board. The extension board must physically close the JTAG chain even if there are no JTAG-configurable components, in order for the complete chain to be closed.

\section{B. Extension Boards}

The initial set of extension boards (Fig. 3) built for E2LP platform contains:

- A board based on Marvell ARMADA 1500 [9] processor for high-end signal processing;

- A board based on NXP LPC processor [10] for simpler programming and control tasks [2]. 

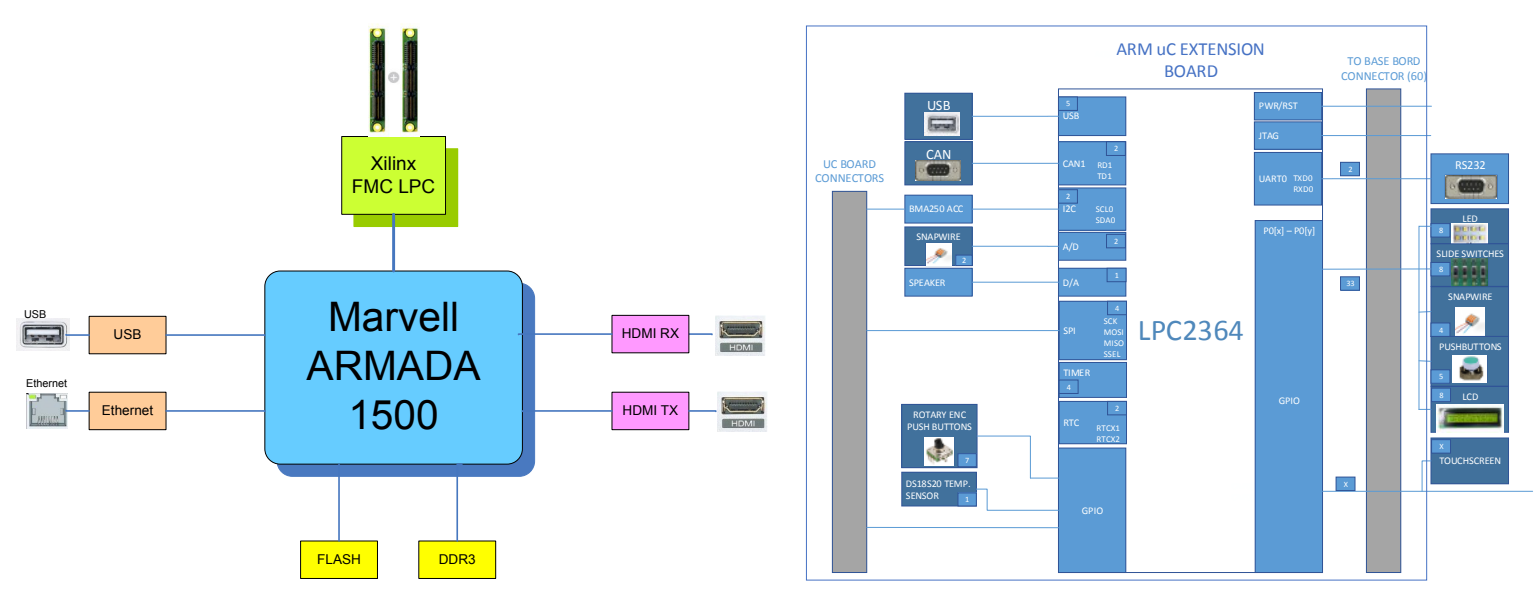

Figure 3. Block diagrams of two extension boards for E2LP platform - ARMADA-based (left) and LPC-based (right)

The extension board based on Marvell ARMADA 1500 has the following functions:

- based on ARM processor, provides the extension to the E2LP platform suitable for highly sophisticated signal processing and execution of real-time software;

- connects to the exterior with USB, LAN and HDMI interfaces and to the Base Board via Mezzanine;

- provides the extension to the E2LP platform suitable for implementing laboratory exercises in the field of digital signal processing, real-time system software, computer networks and system integration.

This extension board is useful for developing laboratory exercises in the field of signal processing, networking and embedded systems design, due to its powerful processor.

For simpler programming assignments, in the first years of computer engineering education, the extension board with a simpler ARM processor is sufficient. It contains:

- LPC2364 microcontroller;

- DS18S20 High-Precision 1-Wire Digital Thermometer;

- LM386 Low Voltage Audio Power Amplifier;

- BMA250 digital accelerometer and I2C;

- Snapwire connector with 8-pins;

- Push-button switches, rotary encoder and LEDs;

- TJA1040 High speed CAN transceiver.

\section{E2LP PLATFORM SOFTWARE}

E2LP platform software allows the student and teacher to use the platform in a convenient way, through an application running on the personal computer (PC). The graphical user interface of the PC application allows the student to download the bit file to FPGA without the need for the platform cable. After the bit file is selected, the software calls Xilinx software to convert bit file to xsvf file ready for download. Afterwards, the transfer to the ARM on board is initiated and performed via USB connection to the board. Finally, FPGA is configured with the transferred xsvf file via JTAG between the ARM and FPGA on the board.

\section{CONCLUSIONS}

The E2LP Base Board together with its extension boards, is working in fully satisfying the main requirement of the E2LP platform - to be used in the complete embedded engineering curriculum and significantly reduce the overhead in engineering education. Extension boards presented in this paper allow achievement of education goals in areas in which the Base Board alone could not be used signal processing, computer networks, system software and application software.

\section{REFERENCES}

[1] I. Kastelan, M. Temerinac, N. Teslic: "E2LP: A Unified Embedded Engineering Learning Platform”, IT System Conference, 2013, pp. 1-4

[2] M. Zagar, N. Frid, J. Knezovic, D. Hofman, M. Kovac, V. Sruk, H. Mlinaric: "Unified, Multiple Target, Computer Engineering Learning Platform", IEEE Global Engineering Education Conference EDUCON, 2014, pp. 926-929

[3] E. Artetxe Gonzalez, A. Setien Gutierrez, J.R. Lopez Benito: "Augmented Reality System for Training, Assistance and Decision Making in Real Time situations in the Embedded Electronic field", $5^{\text {th }}$ Joint Virtual Reality Conference JVRC, 2013, pp. 113-115

[4] R. Schneiderman: "Economy and Shortages Affect the European Job Outlook", IEEE Spectrum, March 2010, Available from (accessed on 2011-12-10) http://spectrum.ieee.org/at-work/tech-careers/economyand-shortages-affect-the-european-job-outlook/0

[5] The European Centre for the Development of Vocational Training (Cedefop): "Skills supply and demand in Europe, Medium-term forecast up to 2020", Publications Office of the European Union, 2010, ISBN 978-92-896-0536-6, Available from (accessed on 201112-10) http://www.cedefop.europa.eu/en/Files/3052_en.pdf

[6] Bureau of Labor Statistics, U.S. Department of Labor, Occupational Outlook Handbook, 2010-11 Edition, Available from (accessed on 2011-12-10) http://www.bls.gov/oco/

[7] M.A. Thompson: "Employment Outlook Asia: Focus on China and India", 2011-04-20, Available from (accessed on 2011-12-10) http://www.goinglobal.com/articles/864/

[8] S. Nooshabadi, J. Garside: "Modernization of teaching in embedded systems design-an international collaborative project", IEEE Transactions on Education, Volume 49, Issue 2, May 2006 pp. 254262

[9] Marvell ARMADA 1500, http://www.marvell.com/digitalentertainment/armada-1500/

[10] NXP LPC2364,

http://www.nxp.com/documents/data_sheet/LPC2364_65_66_67_68.p df 